\title{
Antibiotic-free segregational plasmid stabilization in Escherichia coli owing to the knockout of triosephosphate isomerase (tpiA)
}

\author{
Ram Shankar Velur Selvamani ${ }^{\dagger}$, Maurice Telaar $^{\dagger}$, Karl Friehs ${ }^{*}$ and Erwin Flaschel
}

\begin{abstract}
Background: Segregational stability of plasmids is of major concern for recombinant bacterial production strains. One of the best strategies to counteract plasmid loss is the use of auxotrophic mutants which are complemented with the lacking gene along with the product-relevant ones. However, these knockout mutants often show unwanted growth in complex standard media or no growth at all under uncomplemented conditions. This led to the choice of a gene for knockout that only connects two essential arms of an essential metabolic pathway - the glycolysis.
\end{abstract}

Results: Triosephosphate isomerase was chosen because its knockout will have a tremendous effect on growth on glucose as well as on glycerol. On glycerol the effect is almost absolute whereas on glucose growth is still possible, but with considerably lower rate than usual. This feature is essential because it may render cloning easier. This enzymatic activity was successfully tested as an alternative to antibiotic-based plasmid selection. Expression of a model recombinant $\beta$-glucanase in continuous cultivation was possible with stable maintenance of the plasmid. In addition, the complementation of tpiA knockout strains by the corresponding plasmids and their growth characteristics were tested on a series of complex and synthetic media. The accumulation of methylglyoxal during the growth of tpiA-deficient strains was shown to be a possible cause for the growth disadvantage of these strains in comparison to the parent strain for the Keio Collection strain or the complemented knock-out strain.

Conclusion: Through the use of this new auxotrophic complementation system, antibiotic-free cloning and selection of recombinant plasmid were possible. Continuous cultivation and recombinant protein expression with high segregational stability over an extended time period was also demonstrated.

Keywords: Plasmid, Segregational stability, Triosephosphate isomerase, Auxotrophy, Methylglyoxal, Keio collection, Knockout strain, Continuous cultivation, tpiA

\section{Background}

In 2009 almost one third (45 out of 151) of commercial biopharmaceutical recombinant proteins licensed by FDA and EMEA were produced by cultivation of Escherichia coli [1]. This meant that nearly $30 \%$ of high-value recombinant proteins were produced by means of a plasmid-based expression system. Various other commercial recombinant proteins (e.g. enzymes) or non-protein products (e.g. amino acids) were also obtained with strains of $E$. coli harboring plasmids. Searching the Science Citation Index

\footnotetext{
* Correspondence: karl.friehs@uni-bielefeld.de

${ }^{\dagger}$ Equal contributors

Faculty of Technology, Fermentation Engineering, Bielefeld University, D-33594 Bielefeld, Germany
}

database for "coli recombinant protein" would lead to more than 26,000 results. These facts show the high importance of $E$. coli as an expression system for commercial as well as scientific use.

Expression systems based on E. coli depend in almost all cases on the presence of at least one plasmid, which has to be segregated into dividing cells during growth. Insufficient segregational plasmid stability would render plasmid-based expression systems useless. Therefore, a variety of methods have been developed in order to achieve plasmid stability [2]. The commonly used method consists in adding antibiotics into the cultivation medium and placing genes for antibiotic resistance on the plasmid carrying the target gene. This strategy is widely used in 
research, since most often only low working volumes have to be applied. In industrial biotechnology, the addition of antibiotics can be generally excluded - not only with respect to economic reasons. The elimination of antibiotics from media and waste streams maybe required during downstream processing. Therefore, other methods for plasmid stabilization are in high demand.

A simple strategy in trying to keep a high segregational plasmid stability without adding antibiotics consists in using plasmids of high copy number and expecting that the statistical distribution of plasmids during cell division may always yield cells with at least some plasmids. This works fine as long as the average plasmid copy number is homogeneous [3]. As soon as cells with very low plasmid copy numbers show up preferentially during high growth rates, cells might have not enough time to synthesize plasmids in high copy numbers. Caused by the additional metabolic burden, plasmids may be lost completely, since plasmid-free cells would gain a growth advantage in comparison to plasmid-bearing cells. This would have a drastic influence especially on fed-batchand continuous cultivation processes.

High metabolic burden, basal transcription levels and possible toxic effects of recombinant proteins ask for other methods for stabilizing plasmids. In some cases, changes in the cultivation strategies, mainly by decreasing the specific growth rate, may lead to sufficient plasmid stability. This can be achieved by reducing the cultivation temperature or changing the carbon source of the medium. However, most methods are based on adding some stabilizing elements by genetic engineering.

Some lessons may be learned from nature's strategies to ensure plasmid segregation. Thus, single copy plasmids often show sophisticated stabilizing mechanisms like that based on the par-system which leads to a controlled distribution of plasmids, similar to the highly organized and controlled chromosome distribution in higher organisms. The par-system consists of at least two protein-coding genes and one special site on the plasmid for controlled distribution in the dividing cell [4].

Other systems lead to the post-segregational killing of plasmid-free cells and need the genetic information of a toxin and its corresponding antidote, with the antidote on the target plasmid [5]. Such a combination was recently applied for the development of a Streptomyces based protein expression system [6].

In all these cases, the plasmids may become quite large, leading to a higher metabolic burden. A larger size may also lead to another problem - decreased structural plasmid stability due to stochastic mutations, which would lead to a reduction in productivity, as well. In this regard, the $c c d B / c c d A$ poison-antidote system, modified as a separate-component-stabilization system provides an alternative using very small genetic constructs to provide efficient antibiotic-free maintenance of plasmid in E. coli [7].

Many studies on DNA vaccine production have particularly been interested in alternative plasmid selection mechanisms, due to the need to avoid all kinds of resistance genes or proteins in therapeutic DNA according to safety standards. An RNA based method, using constitutive expression of $s a c B$ as a counter-selectable marker during growth on sucrose was reported to be able to bring about antibiotic-free selection and highly productive fermentation while not being restricted to ColE1 vectors [8].

Another perhaps more elegant way to stabilize the propagation of plasmids, is to destroy the function of an essential gene on the chromosome and place this gene on the plasmid carrying the target genes. This approach requires a strategy to generate competent cells of the now auxotrophic strain. One of the first methods for commercial use was the valS-system [9]. The wild-type gene for valyl-tRNA synthetase (valS) of the host carries a temperature-sensitive mutation, whereas the gene without mutation is placed on a plasmid. For transformation the host is grown at $30^{\circ} \mathrm{C}$. During cultivations at $37^{\circ} \mathrm{C}$ the mutated synthetase on the chromosome loses its function and only cells carrying the valS-harboring plasmid can survive and grow. However, the valS gene is still around on the chromosome and the selection pressure favors a recombination of the mutated vals on the chromosome and the wild type valS on the plasmid. Such a recombination produces revertants with no selection pressure by valS and leads to plasmid instability. One way to reduce the probability of recombination is the complete knockout of the chromosomal gene. In the case of valS this would not allow to obtain viable cells for transformation and, therefore, would not be practicable.

The Operator-Repressor-Titration (ORT) strategy is based on negative regulation of an essential chromosomal gene by an operator sequence allowing the binding of a constitutively expressed repressor protein. In order to allow expression of the essential gene and survive, the cell has to titrate the repressor molecules against a similar operator sequence that may be present in multiple copies on the target plasmid to be maintained [10]. The essential function complementation strategy could also be effective at the RNA level. Recently, an amber nonsense mutation introduced into the essential thyA gene in the chromosome causing thymidine auxotrophy, was overcome by recombinant plasmids carrying a suppressor tRNA, which allowed antibiotic-free plasmid selection and also recombinant luciferase reporter expression in eukaryotic tissues and in tumour cells [11]. The fabI - triclosan system, which is an essential gene- gene product inhibitor combination, was a completely different 
alternative plasmid selection concept. However, the risks associated with the biocide triclosan, the requirement of the selection agent due to an addictive effect, the need to induce and over-express the essential gene marker and plasmid instability in absence of selection were also noted [12]. Another example of antibiotic-free plasmid selection is the strategy involving the essential infA gene coding for translation initiation factor [13].

If an auxotrophy can be overcome by supplements in the medium, competent cells can be prepared and transformed using such a supplemented medium. This may be achieved by knockout of an essential gene for e.g. the synthesis of an amino acid like glycine [14]. In this case, the glyA gene is knocked out in E. coli M15 leading to a auxotrophic strain which can be cultivated on glycine containing media. The $g l y A$ gene is cloned on an expression vector under the control of a constitutive weak promoter. This system has one disadvantage in that glycine-containing media may lead to plasmid-free cells, and many complex industrial media contain glycine.

One solution of this problem would consist in the construction of a knockout strain, the chromosomal knockout of which would still allow at least some growth on complex media. The specific growth rate for a strain harbouring the plasmid containing the knockout gene could generate a selection advantage high enough to keep the plasmid in the cell population.

During the search for a gene which would be appropriate for the application of the auxotrophy-based strategy for plasmid stabilization we have focused on tpiA, the gene for triosephosphate isomerase, a central enzyme in the glycolysis pathway. One of recent great scientific achievements in E. coli research was the establishment of the so-called Keio collection of knockout strains [15]. Using the Keio tpiA knockout strain we present the construction of tpiA-harbouring plasmids leading to E. coli strains bearing plasmids of high segregational stability.

\section{Results}

\section{Screening for an appropriate knockout gene}

As a consequence of biodiesel business, glycerol has evolved as an interesting carbon source for fermentation processes. A closer look on the glycerol metabolism of microorganisms, therefore, is of particular importance. Escherichia coli can utilize glycerol as the sole carbon and energy source. After having been imported through the cytoplasmic membrane by a facilitator protein $(\mathrm{GlpF})$, glycerol can be metabolised on two alternative pathways depending on the growth conditions. One consists of a phosphorylation step by a glycerol kinase (GlpK) to yield L-glycerol-3-phosphate followed by an oxidation step due to the appropriate dehydrogenase (GlpD under aerobic and GlpABC under anoxic conditions) leading to dihydroxyacetone phosphate (DHAP). The alternative pathway consists of an oxidation step by glycerol dehydrogenase (GldA) to yield dihydroxyacetone (DHA) followed by phosphorylation by DHA kinase (DhaK) to give DHAP as well. The kinase-dehydrogenase (GlpK-GlpD/GlpABC) route is the preferred one in E. coli. Accordingly, different genes and their products come into play as there are: glpF $g l p K$ glpD glpABC gldA and dhaK. In addition, the resulting intermediate metabolite DHAP must be fed into the general glycolytic pathway through isomerisation by triosephosphate isomerase (TpiA) as glyceraldehyde-3-phosphate (GA3P). In the absence of this enzyme, DHAP is converted to the toxic compound methylglyoxal [16].

To look for an appropriate gene, four strains with a knockout of each tpiA, glpK, gldA or $g l p F$ were grown on LB-medium in the presence of glycerol as shown in Figure 1.

The knockout of $g l p F$, the gene for the glycerol uptake facilitator protein, has no significant effect on growth. The normal permeability of both plasma and outer membrane may be sufficient for glycerol uptake [17]. When $\operatorname{gld} A$, the glycerol dehydrogenase, is knocked out growth on LB medium is not disturbed, either. Compared with the growth characteristics of the E. coli MG1655 wild type strain, these knockouts seem to be even slightly beneficial. Keio strain $\Delta g l p K$, in which the glycerol kinase is knocked out, does not grow at all, although its effect might be partly circumvented [18]. The second strain showing useful characteristics is that in which the triosephosphate isomerase (tpiA) has been knocked out. In consequence, this gene is considered even more appropriate than $g l p K$ for developing a strategy for plasmid stabilization based on the auxotrophy principle since it represents an enzymatic activity essential also for the glycolysis pathway and not only for glycerol metabolism.

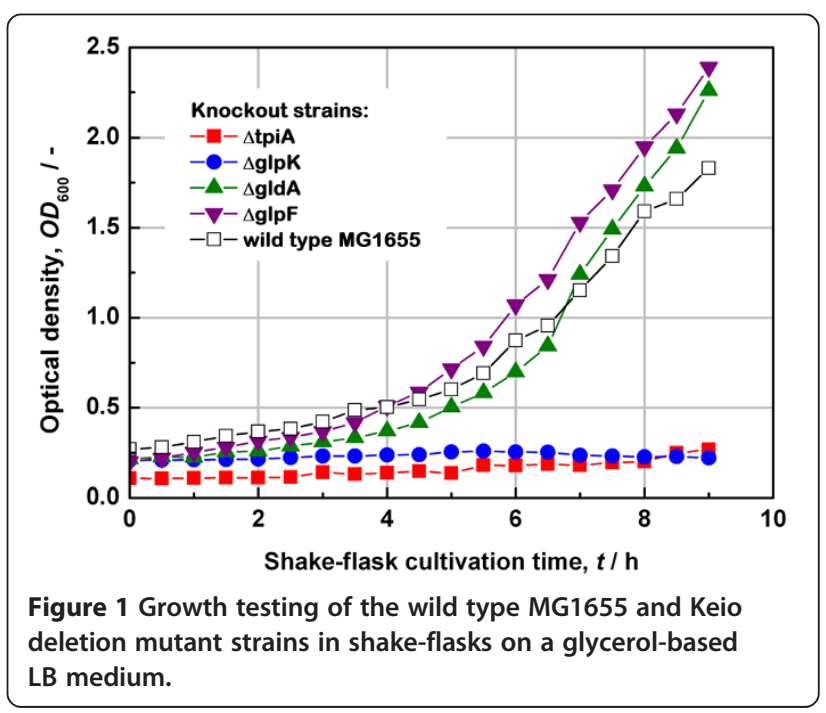




\section{Cloning of tpiA}

For the first construct of the tpiA gene for complementation of tpiA knockout, the wild type promoter- and terminator sequences were chosen. Thus, chromosomal DNA of E. coli MG1655 was isolated and the tpiA region was amplified by PCR using $5^{\prime} \cdot$ taagctggcgctatctgaatcg $\cdot 3^{\prime}$ and $5^{\prime} \cdot$ gatggtacggcagagtgataac $\cdot 3^{\prime}$ as forward and reverse primer, respectively. The amplified tpiA fragment started 150 bp upstream of the ATG start codon and ended $172 \mathrm{bp}$ downstream of the TAA stop codon of the tpiA structural gene. The upstream region started in the yiiQ gene, coding for an unknown conserved protein, including the predicted tpiA promoter [19]. The downstream region reached into the $c d h$ gene including the predicted Rhoindependent tpiA terminator [20]. The resulting PCR fragment of $1090 \mathrm{bp}$ length was cloned into plasmid pJET (Fermentas) and subsequently verified by sequencing. This construct called pJET-tpiA was transformed into the auxotrophic host strain E. coli JW3890-2, CGSC\#: 10805, Keio Collection $(\Delta t p i A)$.

All Keio knockout strains show a special structure in the genome. The knockout locations are found directly behind the ATG start codon followed by the gene for the kanamycin resistance flanked by FRT-sites. The knockouts stop 18 bases in front of the stop codon of the knocked out gene.

In case of the cloned fragment of the tpiA region, there was still an overlap of $153 \mathrm{bp}$ upstream and $195 \mathrm{bp}$ downstream of the structural target gene. This arrangement still involved the risk that a recombination between the knockout location on the chromosome and the tpiA region on a plasmid could occur.

\section{Application of the tpiA knockout in a recombinant production system}

Previous work described the extracellular production of a hybrid bacterial $\beta$-glucanase using plasmid p582 having a size of $6 \mathrm{~kb}$ and a pUC19 origin of replication [21]. A strong constitutive synthetic promoter (CP7) controlled the expression of the $\beta$-glucanase gene [22]. It was followed by a Bacillus-derived signal sequence for periplasmic targeting. At the carboxy terminus a hexahistidine tag was placed for facilitating protein capture and detection. The other important gene on the plasmid p582 was kil coding for the bacteriocin release protein of ColE1 the expression of which would initiate the release of periplasmic proteins into the extracellular space. This gene was under the control of the weak stationary phase promoter of the gene fic. The plasmid contained antibiotic resistance genes against both ampicillin and kanamycin.

Using the plasmid pJET-tpiA as template, the tpiA gene region was amplified with flanking EcoRI ends using the primers $5^{\prime} \cdot$ GGAATTCtaagctggcgctatctg. $3^{\prime}$ and $5^{\prime}$. GGAATTCgatggtacggcagagtg $\cdot 3^{\prime}$. This fragment was then restricted with EcoRI and cloned into the vector p582 at its unique EcoRI site. The result of the cloning procedure is shown in Figure 2. The clones 1 and 4 were subsequently verified with respect to their sequence. Partial sequencing of $\mathrm{pFC} 1$ and $\mathrm{pFC} 4$ revealed that the regions of the cloned tpiA gene were oriented in opposite directions.

The clones number 1 and 4, referred to hereafter as pFC1 and pFC4 respectively, were chosen for further studies and checked for their efficiency to complement the TpiA auxotrophy by transforming competent cells of the strain E. coli JW3890-2. The constructs were found to be functional and the results from growth studies in shake flasks with these plasmid constructs are shown in Figure 3.

As expected, the original Keio tpiA knockout strain (JW3890-2) did not grow in the presence of glycerol as the only carbon source (SGA-Kan). If a complex carbon source was present (LB-Kan), however, some growth was observed. Only the strains complemented with plasmids pFC1 (JW3890-2-pFC1) and pFC4 (JW3890-2-pFC4) showed normal growth behaviour in the presence of glycerol as the only carbon source (SGA-Kan). The strain JW3890-2-pFC1 showed a shorter lag phase while the strain with construct $\mathrm{pFC} 4$ had a relatively longer lag phase. In fact, this phenomenon could be reproduced over multiple trials. Both strains reached equal maximum optical densities $(600 \mathrm{~nm})$ of about 8.0. The media had been supplemented with kanamycin in all three cases. Although, both clones were able to complement the auxotrophy of the tpiA Keio knockout strain, their product expression capabilities differed considerably. Both constructs were studied under batch cultivation conditions in appropriate bioreactors.

\section{Batch fermentation with strain JW3890-2-pFC1}

Batch fermentation using the strain JW3890-2-pFC1 showed that the plasmid pFC1 could complement the gene knockout and enable the strain to grow with glycerol as the sole carbon source. The main results are shown in Figure 4. A biomass concentration of about $8 \mathrm{~g} \mathrm{~L}^{-1}$ was reached after an operating time of $24 \mathrm{~h}$. Extracellular proteins appeared delayed accumulating to $0.4 \mathrm{~g} \mathrm{~L}^{-1}$. The extracellular concentration of $\beta$-glucanase accumulated in parallel to the profile of total extracellular protein, but reached a maximum volumetric activity of just $68 \mathrm{U} \mathrm{mL}^{-1}$. To note again, this is the construct in which the gene tpiA has been cloned in the forward orientation with respect to the kil and $b g l$ gene on the plasmid p582.

\section{Batch fermentation with strain JW3890-2-pFC4}

A similar experiment was carried out with the strain JW3890-2-pFC4. The main results have been gathered 


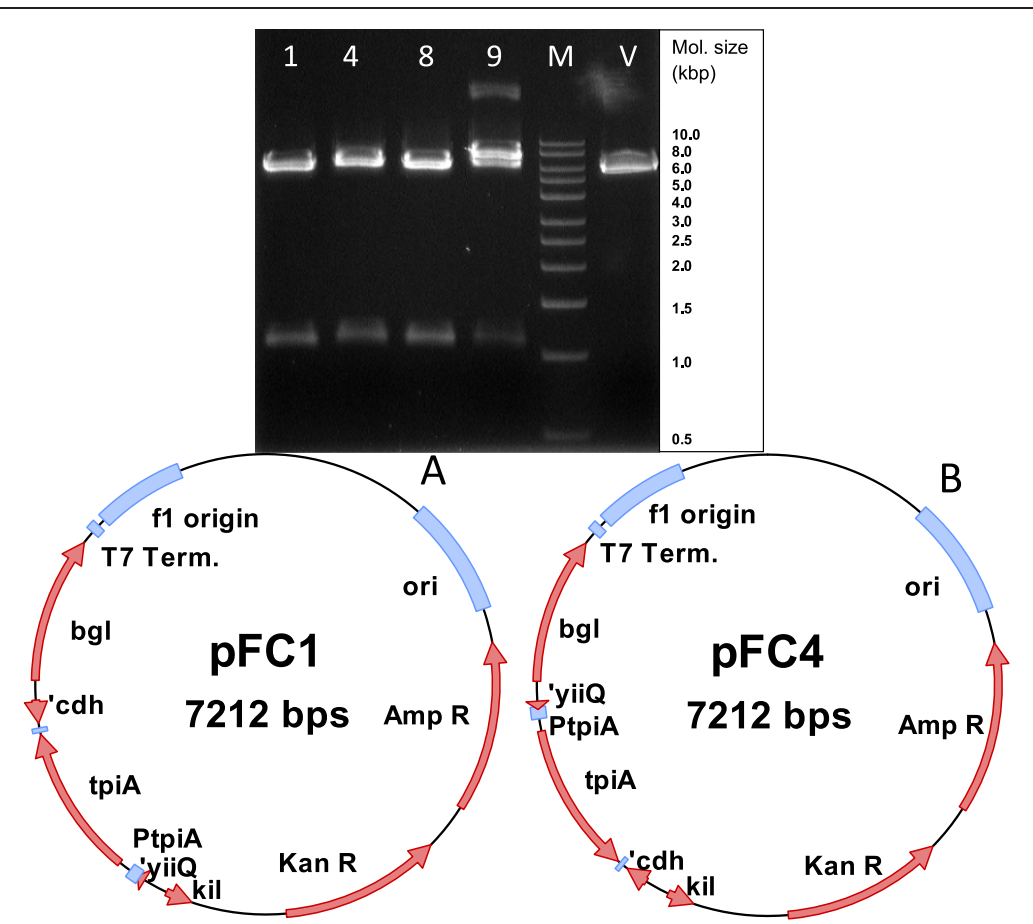

Figure 2 Cloning of complementation cassette into a recombinant protein expression plasmid. Above: EcoRI restriction and screening for positive recombinant clones. Numbers 1, 4, 8, and 9 refer to clones called pFC1, pFC4, pFC8 and pFC9. V refers to the restricted vector p582 as control. M refers to $1 \mathrm{kbp}$ molecular size marker from Plasmid Factory GmbH. Below: Structure of plasmid constructs pFC1 (A) and pFC4 (B). The constructs are each $7 \mathrm{~kb}$ in size and contain the recombinant $\beta$-glucanase expression gene (bgl) and the gene for the bacteriocin release protein (kil). The origin of replication (ori) is based on pUC19. The plasmids carry the beta-lactamase gene for resistance against ampicillin and neomycin phosphotransferase gene for resistance against kanamycin (shown as Amp R and Kan R respectively). The auxotrophy-complementing tpiA gene along with its natural promoter (PtpiA) and terminator sequences has been cloned in the forward orientation in pFC1 and in reverse orientation in $\mathrm{pFC4}$

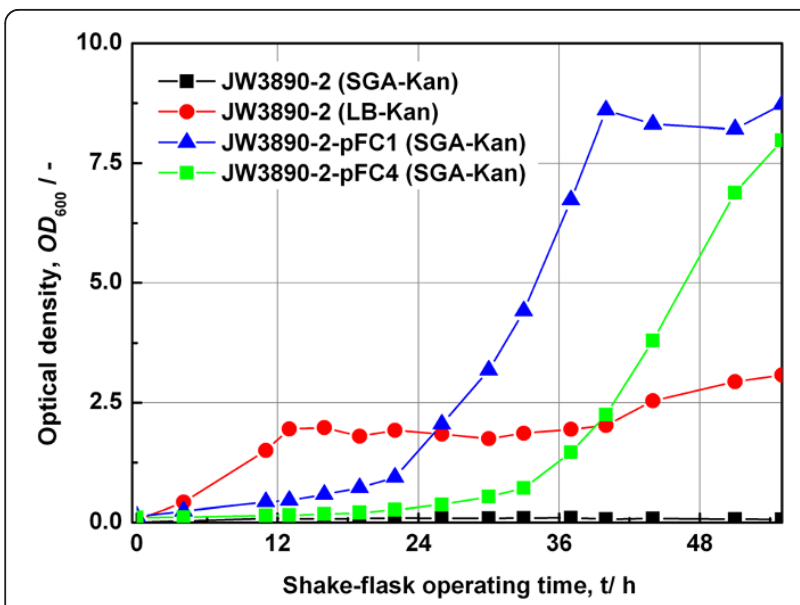

Figure 3 Auxotrophy complementation of tpiA knockout strains. Growth of tpiA knockout strain (JW3890-2) and complemented knockout strains (JW3890-2-pFC1 and JW3890-2-pFC4) were tested in different media based on glycerol only (SGA) or complex carbon sources (LB) in the presence of kanamycin in shake flasks. in Figure 5. From these it was immediately apparent that this construct was able to achieve higher extracellular recombinant enzyme activities as compared to the other clone JW3890-2-pFC1. In fact, the maximum extracellular $\beta$-glucanase activity reached $132 \mathrm{U} \mathrm{mL}^{-1}$ at a somehow lower extracellular total protein concentration

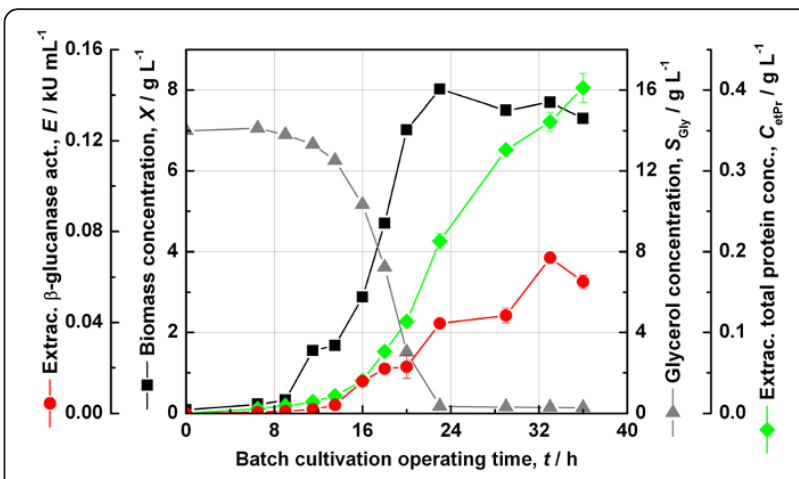

Figure 4 Batch cultivation of E. coli strain JW3890-2-pFC1. The Keio $\Delta$ tpiA knockout strain JW3890-2 complemented with the construct pFC1 was grown in SGA medium in a $2 \mathrm{~L}$ in-house fermenter with $1 \mathrm{~L}$ working volume at $30^{\circ} \mathrm{C}$ without the use of antibiotics. 


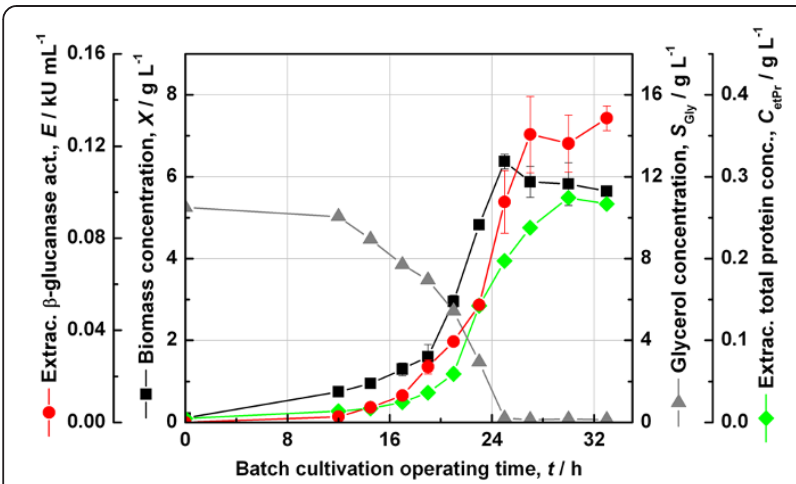

Figure 5 Batch cultivation of E. coli strain JW3890-2-pFC4.

The Keio $\Delta$ tpiA knockout strain JW3890-2 complemented with the construct pFC4 was grown in SGA medium in a $2 \mathrm{~L}$ in-house fermenter with $1 \mathrm{~L}$ working volume at $30^{\circ} \mathrm{C}$ without the use of antibiotics.

of $0.28 \mathrm{~g} \mathrm{~L}^{-1}$. Otherwise the time profiles were similar to those shown in Figure 4.

Since the plasmid pFC4 showed higher extracellular enzyme activity compared to $\mathrm{pFC1}$, it was retained for further studies.

\section{Test of plasmid stabilization with tpiA in continuous culture}

Before starting the chemostat study, the plasmid stability was tested in shake flasks by repeated subculture for over 70 generations and was found to be adequate (data not shown). As a decisive test, a chemostat was set up consisting of a small reactor of $2 \mathrm{~L}$ total volume in which a working volume of $1 \mathrm{~L}$ was maintained. The SGA minimal medium based on glycerol as the only $\mathrm{C}$-source was used. The feed solution contained $20 \mathrm{~g} \mathrm{~L}^{-1}$ glycerol. No antibiotics were added to the media.

At first, the chemostat was operated for an extended period of time for testing the plasmid stability. This test was not only undertaken for the complemented knockout strain JW3890-2-pFC4, but also with the control strain JM109-p582. In the latter case a constant space velocity of $0.1 \mathrm{~h}^{-1}$ was maintained whereas in the former case the space velocity was varied with a profile of $0.2 \mathrm{~h}^{-1}$ for $75 \mathrm{~h}, 0.35 \mathrm{~h}^{-1}$ for $21 \mathrm{~h}, 0.3 \mathrm{~h}^{-1}$ for $19 \mathrm{~h}$, $0.1 \mathrm{~h}^{-1}$ for $77 \mathrm{~h}, 0.25 \mathrm{~h}^{-1}$ for $17 \mathrm{~h}$, and $0.15 \mathrm{~h}^{-1}$ for an additional period of $26 \mathrm{~h}$. This time profile was chosen in order to test the stability under stronger conditions than before. The results for both experiments are gathered in Figure 6. In the absence of antibiotics, the strain JM109-p582 lost its plasmid continually, whereas the plasmid concentration for the strain JW3890-2-pFC4 stabilized at about $10 \mathrm{ng} \mu \mathrm{L}^{-1}$. Thus, the strategy of complementing the auxotrophy based on the triosephosphate isomerase works even in the case of continuous operation. As interesting as that, the expression of extracellular

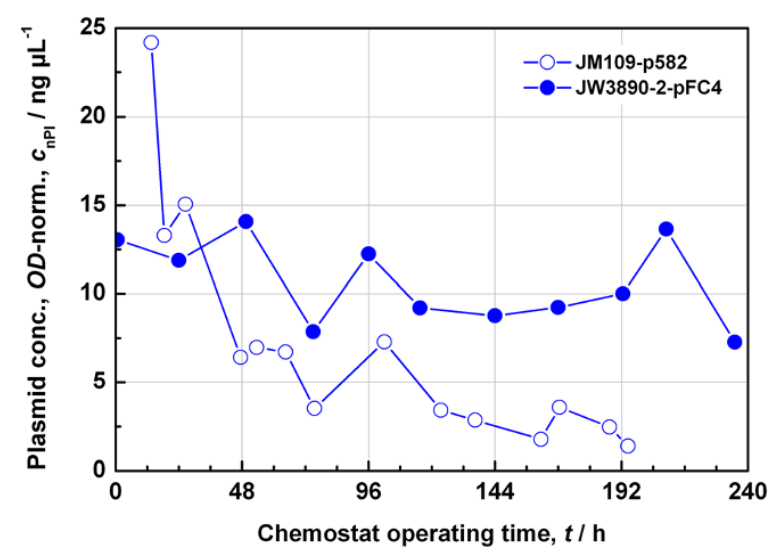

Figure 6 Long-term operation of a chemostat for analysing plasmid maintenance. Plasmid concentrations from the cells were compared for a chemostat with the control culture E. coli JM109p582 and the auxotrophic system E. coli JW3890-2-pFC4 under antibiotic-free conditions.

proteins with $E$. coli seemed to be feasible in continuous operation as well.

The chemostat experiments carried out at different space velocities offer some information about kinetic relationships. The resulting changes in cell density, extracellular $\beta$-glucanase activity, plasmid concentration and the segregational plasmid stability were analysed and the results obtained are shown in Figure 7. The decline of the cell density with higher space velocities revealed a pattern of kinetics far from exponential growth. A similar unfavourable characteristic was found for the extracellular enzyme activity the decline of which was rather linear as a function of space velocity. The maximum volumetric enzyme productivity was fairly low at about $6.5 \mathrm{U} \mathrm{mL}^{-1} \mathrm{~h}^{-1}$. The plasmid stability by replica plating was almost $100 \%$ through the whole process.

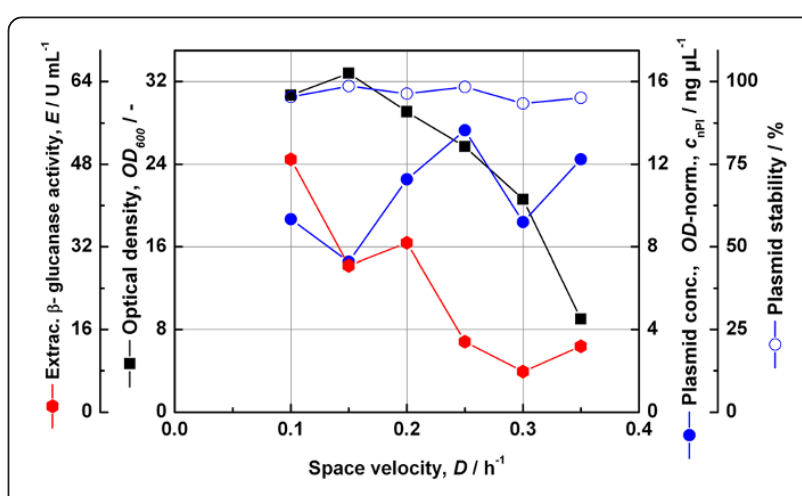

Figure 7 Kinetic behaviour of the expression system JW3890-2pFC4. The auxotrophic complementation system was analysed by means of steady-state chemostat cultivation at different space velocities. 


\section{Construction of a tpiA vector with altered downstream overlap}

In order to decrease the possibility of recombination by the overlaps of the chromosome base sequence and the tpiA region on the plasmid, the original tpiA terminator of pJET-tpiA was replaced by an artificial bidirectional terminator leading to the plasmid pJET-tpiA-Tart. This terminator had the sequence AAAAAAAAAAAGGGGC GAAGCCGCCCCTTTTTTTTT and was cloned downstream of the tpiA gene on pJET-tpiA using the primers shown in Table 1. The portions forming the terminator have been shown in upper case letters.

\section{Growth of the tpiA knockout strain on different media in the absence or presence of the complementing plasmid pJET-tpiA-Tart}

The first step in growth analysis of the tpiA systems was to determine the growth rates of the parent strain for the Keio Collection E. coli BW25113, the Keio deletion mutant JW3890-2, and the plasmid-supplemented mutant strain. In all these experiments the tpiA deficient strain showed lower maximal optical densities and lower growth rates than the parent strain for the Keio Collection and the plasmid supplemented tpiA deficient strain JW3890-2. As an example the growth on the glucose-based HSG medium is shown in Figure 8.

The Keio tpiA knock out strain JW3890-2 reached the stationary phase in HSG medium early. Other media tested were $\mathrm{LB}, \mathrm{LB}+$ ampicillin, $\mathrm{HSG}+$ ampicillin, LB + glycerol, LB + glucose, SGA, SGA + ampicillin, SGA + glucose, SOC and TB, the terrific broth. The composition of the media applied is to be found under Methods. As shown in Figure 9, the tpiA knock out strain JW3890-2 showed no growth in synthetic media. In all other media the strain showed lower growth rates than the parent strain for the Keio Collection and the plasmid bearing strain.

The growth rates of the parent strain for the Keio Collection and the plasmid carrying strain were quite similar. The plasmid supplemented strain was able to grow in synthetic media, whereas the plasmid-free tpiA deletion strain JW3890-2 was not able to grow on synthetic media.

\section{Analysis of plasmid stability}

Because of the fact that the plasmid free tpiA deletion strain could not grow on synthetic media, the experiments

\section{Table 1 Primers for cloning an artificial terminator} behind the tpiA gene on pJET-tpiA

\begin{tabular}{ll}
\hline Primer name & Sequence \\
\hline tpiA Term. lacUV & 5'-CGCCCCTITITTTagcctggggtgcctaatgag-3' \\
tpiA Term. direct & 5'-GCTTCGCCCCTITITTTTTaagcctgtttagccgcttctgc-3' \\
\hline
\end{tabular}

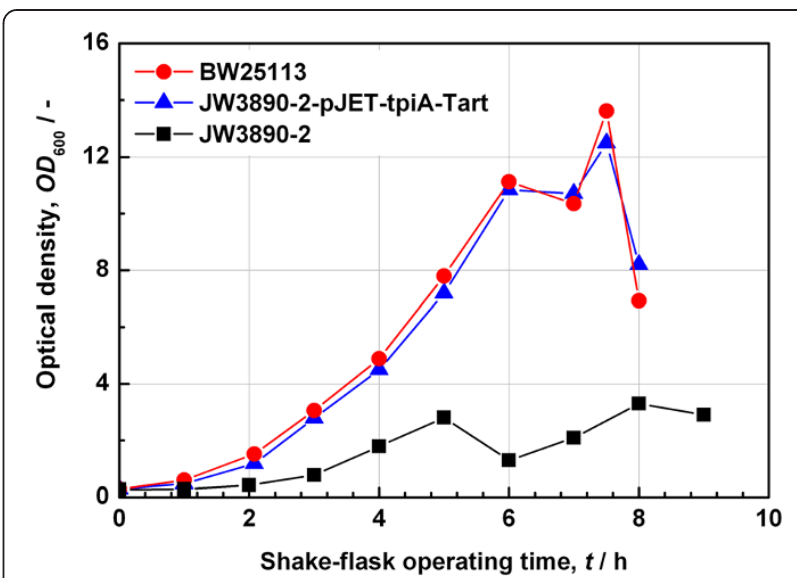

Figure 8 Growth of the parent strain for the Keio Collection BW25113 and JW3890-2 deletion mutant strains. The mutant strains were grown in the absence or presence of the complementing plasmid pJET-tpiA-Tart. The cultivations proceeded in shake flasks on the glucose-based HSG medium.

for antibiotic free selection were performed in SGA medium. For this purpose shake-flask cultivations were carried out in a repetitive fashion by reintroducing $100 \mu \mathrm{L}$ of a final cultivation broth into $50 \mathrm{~mL}$ of fresh medium in shake flasks of $500 \mathrm{~mL}$ for a cultivation period of 1 day. The same procedure was applied to the parent strain for the Keio Collection as well as to the mutant $\Delta t p i A$ Keio strain in the presence of either pJET-tpiA or pJET-tpiATart as complementing plasmid. For each series 13 repeated cultures were followed over a total period of $17 \mathrm{~d}$.

For a cultivation time of $1 \mathrm{~d}$, the parent strain for the Keio Collection strain grew to an optical density of $8.1 \pm$ 0.25 whereas the pJET-tpiA complemented mutant strain reached $12.1 \pm 0.53$, and that complemented with pJETtpiA-Tart grew to an $\mathrm{OD}_{600}$ of $11.3 \pm 0.57$. The data are given with their standard deviations collected over 10 samples. The segregational plasmid stability was tested at the same time and was found to be stable at 98 to $100 \%$.

In addition, the plasmid stability was tested for cultivations in the presence of different media in the absence of ampicillin. Thus, the plasmid maintenance was found to be absolute for the growth of the Keio mutant strain complemented with pJET-tpiA-Tart in the case of the media HSG, LB, LB supplemented with glucose, LB supplemented with glycerol, SGA, SGA supplemented with glucose, SOC as well as TB.

Because of these data it can be expected, that the TpiA deficiency can be used as a selection marker for plasmid stabilization.

Accumulation of methylglyoxal may lead to a toxic effect The reason why the TpiA deficient strain stopped growing at rather low biomass concentrations compared with the wild type or the plasmid-supplemented strain during 

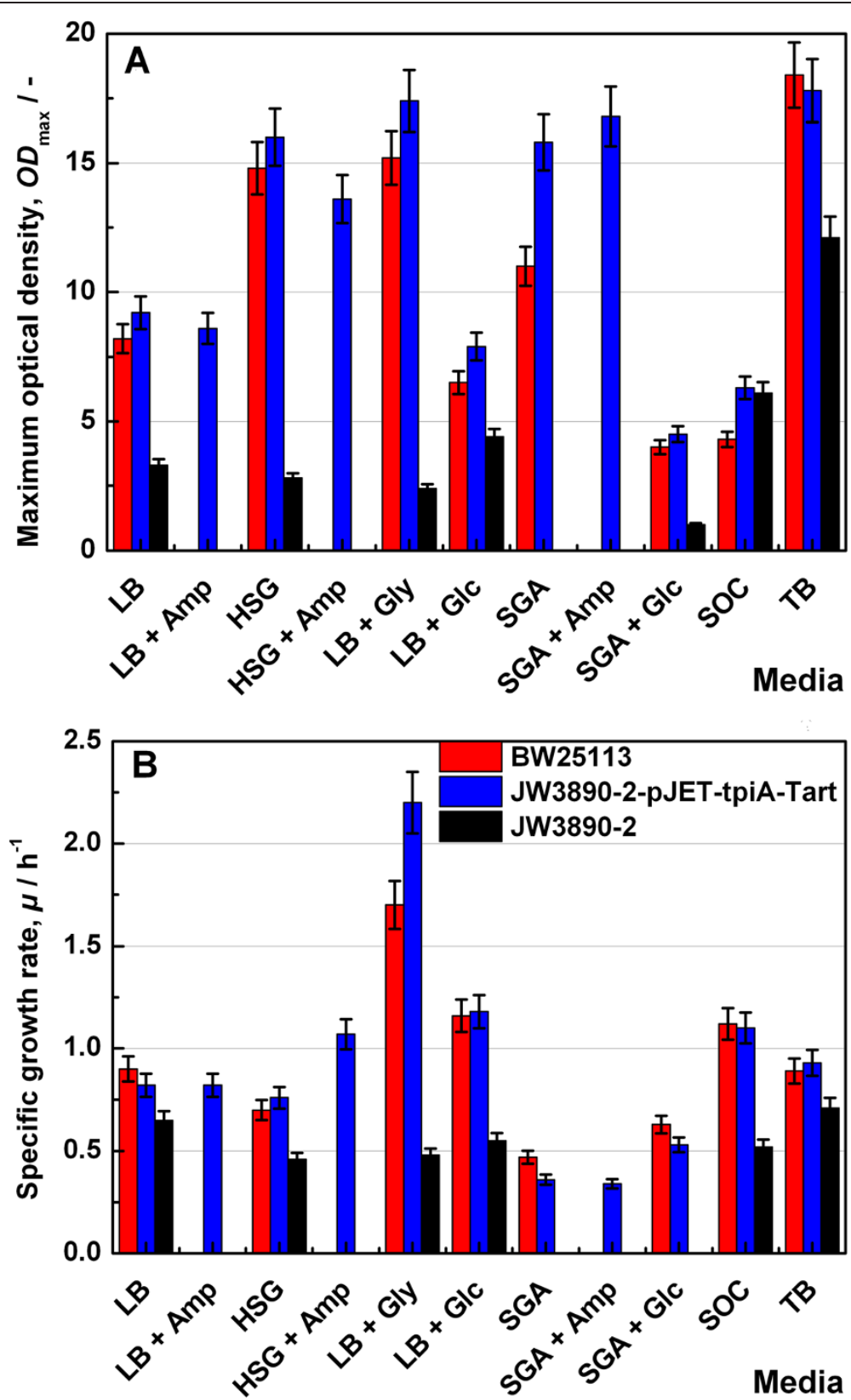

Figure 9 Influence of medium composition on the growth kinetics. Maximum biomass concentration $\left(O D_{600}\right)(\mathbf{A})$ and the specific growth rate $(\mu)$ (B) of E. coli Keio strains: BW25113, knockout mutant in the presence (JW3890-2-pJET-tpiA-Tart), and in the absence (JW3890-2) of the complementing plasmid. The legend applies to both Figure parts (A and B).

cultivation on different media as shown in Figure 9 could be a consequence of DHAP accumulating in the cells. This could in turn lead to methylglyoxal by enzymatic conversion of DHAP by MgsA (methylglyoxal synthase), which should be toxic for the cells. Some experiments were performed in order to test this assumption.

Thus, the tpiA deficient strain JW3890-2 was cultivated in HSG medium overnight. An optical density $\left(\mathrm{OD}_{600}\right)$ of 4.3 was obtained. The culture was centrifuged and the supernatant was sterile filtered. The medium still contained
$10 \mathrm{~g} \mathrm{~L}^{-1}$ glycerol (initial concentration was $14.9 \mathrm{~g} \mathrm{~L}^{-1}$ ). In parallel, a culture of JW3890-2-pJet-tpiA-Tart had been cultivated in HSG medium overnight. The sterile filtered medium was inoculated with JW3890-2-pJet-tpiA-Tart at an initial optical density $\left(\mathrm{OD}_{600}\right)$ of 0.35 . The concentration of glycerol and methylglyoxal were analysed in addition to the optical density along the cultivation process. Figure 10 shows the results. The plasmid supplemented strain grew to an optical density $\left(\mathrm{OD}_{600}\right)$ of 20 within 8 hours by consuming $7 \mathrm{~g} \mathrm{~L}^{-1}$ glycerol. 


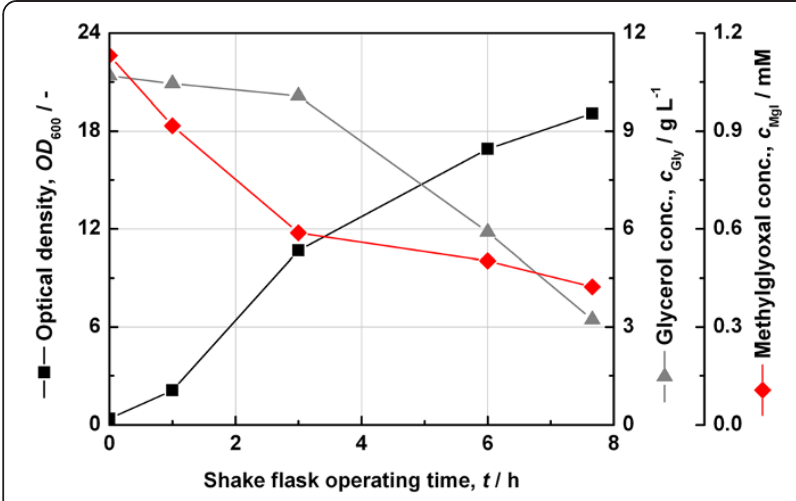

Figure 10 Determination of the toxic effect of methylglyoxal. Shake-flask cultivation of E. coli JW3890-2-pJet-tpiA-Tart on spent HSG medium obtained by prior growth of JW3890-2 on HSG medium.

The data show that methylglyoxal accumulated during the cultivation of the tpiA deficient strain and that it was consumed during the second cultivation on the spent medium, and fell from $1.1 \mathrm{mM}$ to $0.4 \mathrm{mM}$. A methylglyoxal concentration of $0.6 \mathrm{mM}$ is assumed to be toxic for E. coli [16]. Thus, the toxicity of methylglyoxal generated from DHAP may force an auxotrophic effect even in the presence of glucose as the main carbon source.

\section{Antibiotic free cloning}

To test the possibility of antibiotic free cloning with tpiA deficient strains, SGA agar plates were created by adding $15 \mathrm{~g} \mathrm{~L}^{-1}$ agar agar into SGA medium and pouring into petri dishes. The tpiA deficient strain was exposed on these plates and showed no growth. After transforming the plasmid pJET-tpiA-Tart in a chemical competent JW3890-2 strain, colonies appeared in the antibiotic free SGA plates containing the correct plasmid.

\section{Discussion}

The constructs pFC1 and pFC4 contain the auxotrophycomplementing tpiA gene in opposite orientations and some consequences of this difference could be observed from Figures 4 and 5. With respect to growth, it was seen over multiple trials that the strain JW3890-2-pFC1 grew with a slightly shorter lag phase and achieved higher maximum biomass concentrations than JW3890-2-pFC4. Whereas, with respect to the extracellular recombinant $\beta$-glucanase activity, the construct pFC4 clearly returned higher volumetric activity than $\mathrm{pFC1}$ and this difference could also be reproduced over multiple trials. It is common for expression levels to vary between different clones and in this case one possible scenario is that in the case of $\mathrm{pFC1}$, the tpiA gene could be read into the downstream product gene $b g l$, which has the same orientation.

Other experiments conducted to check the secretion achieved with these constructs showed that both the clones $\mathrm{pFC} 1$ and $\mathrm{pFC} 4$ were poor in secretion into extracellular space. The analysis of samples from the stationary phase showed that, the activities in the periplasmic fraction were higher than those in the extracellular fraction. This was in clear contrast to the control strain E. coli JM109-p582, where the reverse was to be seen and the equilibrium was clearly in favour of the extracellular fraction (data not shown). In general, the control strain showed up to 10 times the activity achieved with the auxotrophic strains and thus represented an ideal case. However, since the auxotrophic system offers antibioticfree cultivation, it deserves further development. The question of what could be causing the suboptimal activity of the BRP in the Keio strains remains open at this moment. The general low efficiency of these auxotrophic hosts could be one possible explanation. In this regard, tpiA gene knockouts from the control strain are being developed which should then be able to serve as efficient hosts for the expression and secretion of the recombinant product.

As mentioned under the section Results, the target gene deletion in the Keio strains is restricted to the region between the start codon and the last six codons. The gene deletion in the control strain would be wider and more extensive than in the Keio strain and is thus designed to overcome the risk of recombination of the plasmid-borne tpiA copy with the host genome and loss of auxotrophy. Another potential route to better productivity from the clones would be the expression of tpiA at the minimum required level. This would avoid unnecessarily high rates of transcription and stress in the cells. Therefore, the native P1/P2 promoters for tpiA are being replaced with artificial weak promoters from a synthetic promoter library [23].

Using the Keio knockout strains, a flux from DHAP into the xylulose pathway is not possible due to the deletion of rhamnose metabolism genes [15]. A bypass of DHAP to lactate and pyruvate through methylglyoxal is possible through the glyoxalase I-II pathway [24]. This should also explain the limited growth of cells without the complementation plasmid in complex medium. However, when glycerol is the sole carbon and energy source, the toxicity of methylglyoxal should render this route unfavourable. Moreover, this would only be a partial metabolism and not an energy-efficient pathway to give a growth rate advantage.

\section{Conclusions}

Antibiotic-free recombinant plasmid selection and stabilization in E. coli based on the auxotrophy complementation of the activity of TpiA has been demonstrated. From our observations, we have been able to achieve antibioticfree cloning, selection, expression of a model recombinant product and long-term stability of the plasmid in 
Table 2 List of Escherichia coli strains used

\begin{tabular}{|c|c|c|}
\hline E. coli strain & Genotype & Source \\
\hline BW25113 (parent strain for the Keio Collection) & $\begin{array}{c}\Delta\left(\text { araD-araB)567, } \Delta / a c Z 4787(.: r r n B-3), \text { lambda }{ }^{-}, r p h-1,\right. \\
\Delta(\text { rhaD-rhaB)568, hsdR514 }\end{array}$ & CGSC, Yale University \\
\hline JW3890-2 & $\begin{array}{c}\Delta(a r a D-a r a B) 567, \Delta / a c Z 4787\left(: \text { :rrnB-3), lambda }{ }^{-}, r p h-1,\right. \\
\Delta(\text { rhaD-rhaB)568, hsdR514 AtpiA778::kan }\end{array}$ & CGSC, Yale University \\
\hline MG 1655 & $\mathrm{~F}^{-}$, lambda ${ }^{-}$, rph-1 & $\mathrm{DSMZ}$ \\
\hline JM109 & $\begin{array}{c}\text { endA1, recA1, gyrA96, thi, hsdR17 (rk-, mk+), relA1, supE44, } \\
\Delta\left(\text { (lac-proAB), [F' traD36, proAB, laql }{ }^{\mid} Z \Delta M 15\right]\end{array}$ & Promega \\
\hline
\end{tabular}

continuous culture. The growth advantage shown by the plasmid-complemented strain even under non-selective conditions makes this system particularly attractive for large-scale industrial processes.

\section{Methods}

The list of strains of E. coli used in this study is shown in Table 2.

All the modifications in the knockout strain other than the tpiA deletion are already present on the parent strain E. coli BW25113. The modifications in the arabinose, lactose or rhamnose metabolic genes do not have any consequences for the current experiments reported with this strain.

The plasmids applied for the experiments described are gathered in Table 3.

The media applied for growth experiments with $E$. coli strains are shortly explained prior to presenting their exact composition in Tables 4 and 5 for convenience.

SGA media are fully synthetic ones. All solutions were autoclaved separately and combined as explained in Table 4. Depending on the preferred carbon source either glycerol or glucose was added. For batch fermentations with SGA, water and carbon source were autoclaved in situ, while the other components were sterilized separately and added to the reactor. When the medium was complete, the $\mathrm{pH}$ was found to be around 6.5. This was then corrected to a $\mathrm{pH}$ of 7 through addition of sterile $\mathrm{NaOH}$ before inoculation. For the strain JM109, sterilefiltered thiamine solution was added at a final concentration of $0.01 \mathrm{~g} \mathrm{~L}^{-1}$ due to its auxotrophy as given in the genotype.

The HSG medium is a buffered complex rich medium with glycerol as carbon source. The $\mathrm{K}_{2} \mathrm{HPO}_{4} / \mathrm{KH}_{2} \mathrm{PO}_{4}$ solution was autoclaved separately. The $\mathrm{pH}$ was adjusted to 7.4. Its composition is explained in Table 5 .

Other media like LB (Lysogeny Broth), TB (Terrific Broth) and SOC (Super Optimal broth with Catabolite repression) were prepared according to standard laboratory protocols.

\section{Cultivation}

\section{Shake flask cultivation}

All cultivations were performed in a working volume of $50 \mathrm{~mL}$ in shake flasks of a total volume of $500 \mathrm{~mL}$ equipped with baffles (Schott, Germany). The rotary shaker LS-X (Kühner, Switzerland) had a rotating frequency of $120 \mathrm{~min}^{-1}$ and an eccentricity (diameter) of $50 \mathrm{~mm}$. The cultivation was carried out at a temperature of $30^{\circ} \mathrm{C}$.

\section{Bioreactor}

The bioreactor cultivations were carried out in a $2 \mathrm{~L}$ inhouse fermenter with a working volume of $1 \mathrm{~L}$. The reactor had a height of $280 \mathrm{~mm}$ and a diameter of $94.4 \mathrm{~mm}$. The impeller diameter measured $46 \mathrm{~mm}$ and the impeller blades measured $12 \mathrm{~mm}$ on each side. A total of 3 impellers with 6 blades each ensured good mixing for homogeneous conditions. Moreover, the vessel contained 4 baffles with a height of $260 \mathrm{~mm}$ and width of $8 \mathrm{~mm}$ each.

\section{Batch cultivation}

Water and glycerol for the SGA medium were sterilized in situ along with the fermenter, while the remaining components were sterilized separately and added under sterile conditions. After adjusting initial $\mathrm{pH}$ and saturating the medium with oxygen, the fermenter was inoculated from an overnight shake flask culture. Agitation

Table 3 List of plasmids used

\begin{tabular}{|c|c|c|}
\hline Plasmid & Features/Genes & Source \\
\hline p582 & rep $(\mathrm{pUC19})$, bla $\left(A m p^{\mathrm{R}}\right), n p t\left(\mathrm{Kan}^{\mathrm{R}}\right), \mathrm{P}_{\mathrm{CP} 7}-b g l, \mathrm{P}_{\mathrm{fic}}-k i l, \mathrm{~T} 7$ terminator, multiple cloning site & {$[21]$} \\
\hline pJET 1.2 & rep $(\mathrm{pMB} 1)$, bla $\left(\mathrm{Amp}^{\mathrm{R}}\right)$, eco47IR, $\mathrm{P}_{\text {lacuv5, }} \mathrm{T7}$ promoter, multiple cloning site, insertion site & Fermentas \\
\hline pJET-tpiA & rep $(\mathrm{pMB} 1)$, bla $\left(\mathrm{Amp}^{\mathrm{R}}\right)$, eco47IR, $\mathrm{P}_{\text {lac } \cup v 5}, \mathrm{T7}$ promoter, residual multiple cloning site, tpiA expression cassette & This work \\
\hline $\mathrm{pFC1}$ & rep (pUC19), bla $\left(A m p^{R}\right), n p t\left(K_{a n}^{R}\right), P_{C P 7}-b g l, P_{f i c}-k i l, T 7$ terminator, tpiA expression cassette in forward orientation & This work \\
\hline $\mathrm{pFC4}$ & rep $(p \cup C 19), b l a\left(A m p^{R}\right), n p t\left(K_{a n}{ }^{R}\right), P_{C P 7}-b g l, P_{f i c}-k i l, T 7$ terminator, tpiA expression cassette in reverse orientation & This work \\
\hline pJET-tpiA-Tart & rep $(\mathrm{pMB} 1)$, bla $\left(\mathrm{Amp}^{\mathrm{R}}\right)$, eco47IR, T7 promoter, residual multiple cloning site, tpiA expression cassette, artificial terminator & This work \\
\hline
\end{tabular}


Table 4 Composition of the SGA medium

\begin{tabular}{|c|c|c|c|c|}
\hline Solution name & Volume fraction/\% & Component & Solution concentration $/ \mathrm{g} \mathrm{L}^{-1}$ & Medium concentration/g $\mathrm{L}^{-1}$ \\
\hline \multirow[t]{8}{*}{ Micronutrient solution 500x } & 0.2 & $\mathrm{FeCl}_{3} \cdot 6 \mathrm{H}_{2} \mathrm{O}$ & 5.4 & 0.0108 \\
\hline & & $\mathrm{ZnSO}_{4} \cdot 7 \mathrm{H}_{2} \mathrm{O}$ & 1.38 & 0.00276 \\
\hline & & $\mathrm{MnSO}_{4} \cdot \mathrm{H}_{2} \mathrm{O}$ & 1.85 & 0.0037 \\
\hline & & $\mathrm{CoSO}_{4} \cdot 7 \mathrm{H}_{2} \mathrm{O}$ & 0.56 & 0.00112 \\
\hline & & $\mathrm{CuCl}_{2}$ & 0.17 & 0.00034 \\
\hline & & $\mathrm{H}_{3} \mathrm{BO}_{3}$ & 1 & 0.002 \\
\hline & & $\mathrm{Na}_{2} \mathrm{MoO}_{4} \cdot 2 \mathrm{H}_{2} \mathrm{O}$ & 2.5 & 0.005 \\
\hline & & Citric acid hydrate & 5 & 0.01 \\
\hline \multirow[t]{4}{*}{ Salt solution $10 x$} & 10 & $\mathrm{~K}_{2} \mathrm{HPO}_{4}$ & 135.3 & 13.53 \\
\hline & & $\mathrm{KH}_{2} \mathrm{PO}_{4}$ & 66.2 & 6.62 \\
\hline & & Citric acid hydrate & 18.4 & 1.84 \\
\hline & & EDTA & 0.08 & 0.008 \\
\hline Glycerol solution & 1 & Glycerol & 1,000 & 10 \\
\hline Glucose solution & 10 & Glucose & 195.6 & 19.56 \\
\hline Ammonium sulphate solution & 1.2 & $\left(\mathrm{NH}_{4}\right)_{2} \mathrm{SO}_{4}$ & 500 & 6 \\
\hline $\mathrm{MgSO}_{4}$ solution & 0.24 & $\mathrm{MgSO}_{4}$ & 250 & 0.6 \\
\hline
\end{tabular}

was carried out at a stirrer speed of $800 \mathrm{rpm}$ and aeration was achieved with an air space velocity of $1 \mathrm{vvm}$. The process temperature was maintained at $37^{\circ} \mathrm{C}$ for the control strain E. coli JM109-p582 and $30^{\circ} \mathrm{C}$ for the auxotrophic strains, since the latter showed inclusion body formation due to stress (data not shown) when grown at $37^{\circ} \mathrm{C}$. The $\mathrm{pH}$ was automatically maintained at 7.0 using $10 \%$ orthophosphoric acid and $2 \mathrm{M}$ sodium hydroxide as correction solutions. The process was monitored by a ADAM-4060 relay output module (Advantech Ltd., USA) and run by using DASYLAB 6.0 software (National Instruments Service GmbH, Germany).

\section{Continuous cultivation}

The chemostat process was started as a batch fermentation, and upon reaching the end of exponential phase, the feed and outlet pumps were switched on. The desired space velocity (dilution rate) was set by the flow rate of the medium. The feed medium supplied from a

Table 5 Composition of the HSG medium

\begin{tabular}{cc}
\hline Medium component & Medium concentration $/ \mathbf{g ~ L}^{\mathbf{1}}$ \\
\hline Soy peptone & 13.5 \\
Yeast extract & 7 \\
Glycerol & 14.9 \\
$\mathrm{NaCl}$ & 2.5 \\
$\mathrm{~K}_{2} \mathrm{HPO}_{4}$ & 2.3 \\
$\mathrm{KH}_{2} \mathrm{PO}_{4}$ & 1.5 \\
$\mathrm{MgSO}_{4} \cdot \mathrm{H}_{2} \mathrm{O}$ & 0.14 \\
\hline
\end{tabular}

$40 \mathrm{~L}$ reservoir had the same composition as the medium for batch but with $20 \mathrm{~g} \mathrm{~L}^{-1}$ glycerol. The culture volume in the reactor was maintained at a constant level by operating the inlet pump at the exact flow rate required whereas the outlet pump was operated at twice the flow rate, with the suction pipe entrance positioned at a fixed depth corresponding to a liquid working volume of $1 \mathrm{~L}$. Stably maintained levels of dissolved oxygen (DO) in the reactor medium, $\mathrm{CO}_{2}$ content in the exit air and optical density of the culture indicated a steady state. Any change in the feed flow rate was followed by allowing the system to stabilize by passing at least three to four culture volumes through the reactor before a new steady state was assumed.

\section{Measurement of growth}

Prior to each cultivation experiment a single colony was transferred from an agar plate into the appropriate medium and cultivated overnight. The pre-culture media were exactly the same as the media used in the subsequent cultivation experiments.

For the main cultivation the initial optical density at a wavelength of $600 \mathrm{~nm}\left(O D_{600}\right)$ was adjusted to 0.1 . Over the cultivation process samples were taken and the optical density was measured against the cultivation medium. If required, samples were diluted with fresh cultivation medium.

\section{Determination of the specific growth rate}

The specific growth rate $(\mu)$ was determined by means of the standard method [25]. 


\section{Plasmid stability}

The plasmid stability was determined by plating out appropriately diluted samples on normal LB agar plates prepared without antibiotics and incubating at $37^{\circ} \mathrm{C}$ for at least $15 \mathrm{~h}$. The colonies were then transferred onto an LB agar plate containing $200 \mathrm{mg} \mathrm{L}^{-1}$ ampicillin followed by incubation at $37^{\circ} \mathrm{C}$ for at least $15 \mathrm{~h}$. The ratio of the number of colonies counted on plates prepared in the presence of antibiotics to that observed in the absence of antibiotics was interpreted as plasmid stability.

For measurements of the plasmid content in the samples, a fixed volume of culture broth $(2 \mathrm{~mL})$ was used for plasmid isolation by the Wizard Plus SV DNA Purification System (Promega, USA). The elution of the plasmid from the column was carried out into a constant volume $(50 \mu \mathrm{L})$ of nuclease-free water (Promega, USA). The plasmid DNA concentration was measured by means of the Nanodrop spectrophotometer (Peqlab Biotechnologie $\mathrm{GmbH}$, Germany), and the value was normalised to the optical density of the culture sample.

\section{Determination of methylglyoxal concentration}

Methylglyoxal was quantitatively determined according to the method described by Cooper [26].

\section{Determination of glycerol concentration}

Culture samples were centrifuged to separate the cells. Glycerol concentration in the supernatant was measured by HPLC using a Nucleogel sugar $810 \mathrm{H}$ cation exchange column (Macherey-Nagel GmbH, Germany).

\section{Assay for $\beta$-Glucanase activity}

The recombinant protein expression was analysed with an activity test for endo-1,3-1,4- $\beta$-glucan-glucanohydrolases. Substrates used were either lichenan (Roth, Germany) or barley $\beta$-glucan (Megazyme, Ireland) both of which have very similar properties. The substrate contains $70 \% \beta-1,4-$ and $30 \% \beta-1,3$-bonds.

The substrate solution in $40 \mathrm{mM}$ sodium acetate buffer [27] was incubated with samples containing the enzyme to be assayed for $20 \mathrm{~min}$. The reducing groups released were analysed by the method based on dinitrosalicylic acid by following the absorbance of light at a wavelength of $530 \mathrm{~nm}\left(O D_{530}\right)$. This was compared to the maximum value expected for the particular substrate at complete conversion, which can be obtained practically by applying an excess enzyme activity and incubating for $2 \mathrm{~h}$. The final enzyme activity was obtained as a volumetric activity taking into account the particular enzyme kinetics [27]. One unit is defined as the enzyme activity that releases an equivalent amount of $1 \mu \mathrm{mol}$ glucose residues per min at a temperature of $50^{\circ} \mathrm{C}$ and a $\mathrm{pH}$ 5.6.

\section{Cloning}

All cloning experiments were carried out according to standard methods [28].

\section{Sequencing}

Potential clones were verified by sequencing the cloned region using corresponding sequencing primers. Cyclesequencing was performed at the Centre for Biotechnology (CeBiTec) (Bielefeld University, Germany). A BigDye ${ }^{\circ}$ terminator version 3.1 chemistry was used for the PCR in a GeneAmp PCR System 9700 (both Applied Biosystems, Germany). The fragments of various lengths were then separated by capillary electrophoresis and the fluorescence signal of each base converted to their corresponding digital data by a 96-well 3730xl DNA Analyzer (Applied Biosystems, Germany).

\section{Competing interests}

The authors declare that they have no competing interests.

\section{Authors' contributions}

RSVS and MTE designed and performed the experiments, analyzed and interpreted the data. KF and EF wrote the manuscript. KF and EF conceived the study and supervised the research. All authors read and approved the final manuscript.

\section{Acknowledgements}

The authors would like to thank Eberhard "Ebson" Wünsch for technical assistance in the lab. RSVS thanks the Deutscher Akademischer Austauschdienst (DAAD) for financial support through providing a PhD scholarship.

We acknowledge support of the publication fee by Deutsche

Forschungsgemeinschaft and the Open Access Publication Funds of Bielefeld University.

Received: 23 January 2014 Accepted: 10 April 2014

Published: 21 April 2014

\section{References}

1. Ferrer-Miralles N, Domingo-Espín J, Corchero UL, Vázquez E, Villaverde A: Microbial factories for recombinant pharmaceuticals. Microb Cell Fact 2009, 8:17

2. Friehs K: Plasmid copy number and plasmid stability. In New Trends and Developments in Biochemical Engineering, Volume 86. Edited by Scheper T. Berlin Heidelberg: Springer-Verlag; 2004:47-82.

3. Summers D: The Biology of Plasmids. Oxford: Blackwell-Science; 1996.

4. Easter CL, Sobecky PA, Helinski DR: Contribution of different segments of the par region to stable maintenance of the broad-host-range plasmid RK2. J Bacteriol 1997, 179:6472-6479.

5. Zielenkiewicz U, Ceglowski P: Mechanisms of plasmid stable maintenance with special focus on plasmid addiction systems. Acta Biochim Pol 2001, 48:1003-1023.

6. Sevillano L, Diaz M, Santamaria Rl: Stable expression plasmids for Streptomyces based on a toxin-antitoxin system. Microb Cell Fact 2013, 12:39.

7. Szpirer CY, Milinkovitch MC: Separate-component-stabilization system for protein and DNA production without the use of antibiotics. Biotechniques 2005, 38:775-781.

8. Luke J, Carnes AE, Hodgson CP, Williams JA: Improved antibiotic-free DNA vaccine vectors utilizing a novel RNA based plasmid selection system. Vaccine 2009, 27:6454-6459.

9. Nilsson J, Skogman SG: Stabilization of Escherichia coli tryptophanproduction vectors in continuous cultures: a comparison of three different systems. Nat Biotechnol 1986, 4:901-903.

10. Cranenburgh RM, Lewis KS, Hanak JAJ: Effect of plasmid copy number and lac operator sequence on antibiotic-free plasmid selection by 
operator-repressor titration in Escherichia coli. J Mol Microbiol Biotechnol 2004, 7:197-203.

11. Marie C, Vandermeulen G, Quiviger M, Richard M, Préat V, Scherman D: pFARs, Plasmids free of antibiotic resistance markers, display high-level transgene expression in muscle, skin and tumour cells. J Gene Med 2010, 12:323-332. doi:10.1002/jgm.1441

12. Goh S, Good L: Plasmid selection in Escherichia coli using an endogenous essential gene marker. BMC Biotechnol 2008, 8:61. doi:10.1186/1472-6750-8-61.

13. Hägg P, de Pohl JW, Abdulkarim F, Isaksson LA: A host/plasmid system that is not dependent on antibiotics and antibiotic resistance genes for stable plasmid maintenance in Escherichia coli. J Biotechnol 2004, 111:17-30.

14. Vidal L, Pinsach J, Striedner G, Caminal G, Ferrer P: Development of an antibiotic-free plasmid selection system based on glycine auxotrophy for recombinant protein overproduction in Escherichia coli. J Biotechnol 2008, 134:127-136.

15. Baba T, Ara T, Hasegawa M, Takai Y, Okumura Y, Baba M, Datsenko KA, Tomita M, Wanner BL, Mori H: Construction of Escherichia coli K-12 in-frame, single-gene knockout mutants: the Keio collection. Mol Syst Biol 2006, 2:2006.0008. doi:10.1038/msb4100050.

16. Tötemeyer S, Booth NA, Nichols WW, Dunbar B, Booth IR: From famine to feast: the role of methylglyoxal production in Escherichia coli. Mol Microbiol 1998, 27:553-562.

17. Truniger V, Boos W: Glycerol uptake in Escherichia coli is sensitive to membrane lipid composition. Res Microbiol 1993, 144:565-574.

18. Applebee MK, Joyce AR, Conrad TM, Pettigrew DW, Palsson B $\varnothing$ : Functional and metabolic effects of adaptive glycerol kinase (GLPK) mutants in Escherichia coli. J Biol Chem 2011, 286:23150-23159.

19. Mendoza-Vargas A, Olvera L, Olvera M, Grande R, Vega-Alvarado L, Taboada B, Jimenez-Jacinto V, Salgado H, Juárez K, Contreras-Moreira B, Huerta AM, Collado-Vides J, Morett E: Genome-wide identification of transcription start sites, promoters and transcription factor binding sites in E. coli. PLoS One 2009, 4(10):e7526. doi:10.1371/journal.pone.0007526.

20. Keseler IM, Collado-Vides J, Santos-Zavaleta A, Peralta-Gil M, Gama-Castro S, Muñiz-Rascado L, Bonavides-Martinez C, Paley S, Krummenacker M, Altman T, Kaipa P, Spaulding A, Pacheco J, Latendresse M, Fulcher C, Sarker M, Shearer AG, Mackie A, Paulsen I, Gunsalus RP, Karp PD: EcoCyc: a comprehensive database of Escherichia coli biology. Nucleic Acids Res 2011, 39:D583-D590.

21. Beshay U, Miksch G, Friehs K, Flaschel E: Integrated bioprocess for the production and purification of recombinant proteins by affinity chromatography in Escherichia coli. Bioproc Biosyst Eng 2009, 32:149-158.

22. Borriss $\mathrm{R}$, Olsen $\mathrm{O}$, Thomsen $\mathrm{KK}$, von Wettstein D: Hybrid bacillus endo-(1-3,1-4)-beta-glucanases: construction of recombinant genes and molecular properties of the gene products. Carlsberg Res Commun 1989, 54:41-54.

23. Jensen PR, Hammer K: The sequence of spacers between the consensus sequences modulates the strength of prokaryotic promoters. Appl Environ Microbiol 1998, 64:82-87.

24. Ferguson GP, Tötemeyer S, MacLean MJ, Booth IR: Methylglyoxal production in bacteria: suicide or survival? Arch Microbiol 1998, 170:209-219.

25. Stanbury PF, Whitaker A, Hall SJ: Principles of Fermentation Technology. Oxford: Butterworth Heinemann; 1995.

26. Cooper RA, Anderson A: The formation and catabolism of methylglyoxal during glycolysis in Escherichia coli. FEBS Lett 1970, 11:273-276.

27. Beshay U, Miksch G, Flaschel E: Improvement of a beta-glucanase activity test by taking into account the batch reactor balance of the test system. Bioproc Biosyst Eng 2007, 30:251-259.

28. Sambrook J, Fritsch EF, Maniatis T: Molecular Cloning: A Laboratory Manual. Cold Spring Harbor: Cold Spring Harbor Laboratory Press; 1989.

doi:10.1186/1475-2859-13-58

Cite this article as: Velur Selvamani et al:: Antibiotic-free segregational plasmid stabilization in Escherichia coli owing to the knockout of triosephosphate isomerase (tpiA). Microbial Cell Factories 2014 13:58.

\section{Submit your next manuscript to BioMed Central and take full advantage of:}

- Convenient online submission

- Thorough peer review

- No space constraints or color figure charges

- Immediate publication on acceptance

- Inclusion in PubMed, CAS, Scopus and Google Scholar

- Research which is freely available for redistribution

Submit your manuscript at www.biomedcentral.com/submit
C Biomed Central 